\title{
THE DATA ACCESS AND TRANSFER SUPPORT IN A LOCAL
}

HETEROGENEOUS NETWORK (HMINET)

R. Popescu-Zeletin

Hahn-Meitner-Institut für Kernforschung Berlin GmbH

Department of Computer Science and Electronics

Working Group: HMI-Computer Network

Glienicker StraBe 100, D-1000 Berlin 39, West-Germany

\section{Abstract}

The following paper presents a local heterogeneous network, the HMINET. The adopted software architecture identifies a data access and transfer level. The higher level applications afford a mechanism for data access and transfer to alleviate the effects of the incompatibilities and the differences between the data management systems. The paper describes the major problems and design decisions we adopted for the Data Access and Transfer System (DATS).

\section{Introduction}

A cooperation of the Hahn-Meitner-Institut for Nuclear Research and Siemens AG, sponsored by the German Ministry for Research \& Technology (BMFT), has led to the development of the computer network under consideration. This development has achieved a star-shaped computer network - the HMINET, which has been operating since 1976 .

The network [8] connects a large number of processes control computers of different manufacturers and three time-sharing mainframes (SIEMENS $7.760,7.755$ and 7.748 ) via a central switching node (SIEMENS 330) using high speed data lines (up to $200 \mathrm{~kb} / \mathrm{s}$ ). The process control computers serve as data acquisition devices for nuclear physics and radiation chemistry experiments. A more complex connection structure is planned, especially the installation of an interconnecting link to an external network in Berlin (BERNET), which will connect research institutes and university computing centers.

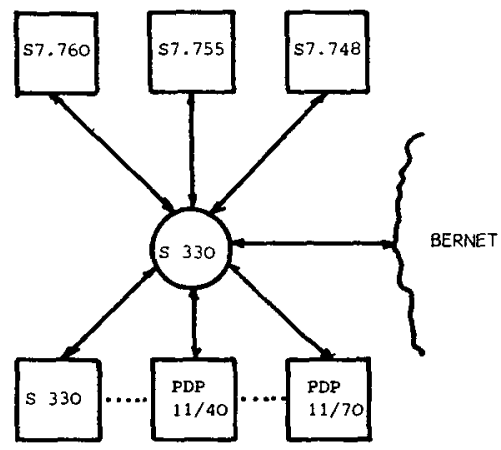

An overview of the network software structure is given in the following figure:

HOST A

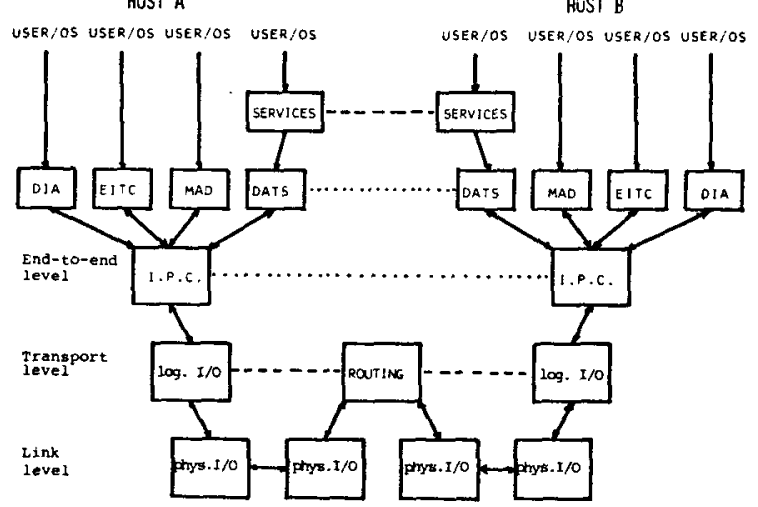

On the link and transport level the SIEMENSNEA2-protocol is used for a datagram service. The software in the end-to-end level provides the interprocess communication facilities.

On the application level there are several basic functions such as

DIA: permitting remote terminal access, defined as access to the time-sharing hosts from all terminals connected to the network.

EITC: permitting an extended inter-task communication between programs running on different hosts.

MAD: performing the control functions in the network

(measurement, administration and display).

DATS: Data access and transfer system for services as: distributed graphics system, electronic mail, distributed data base, remote job entry, distributed data management system. 


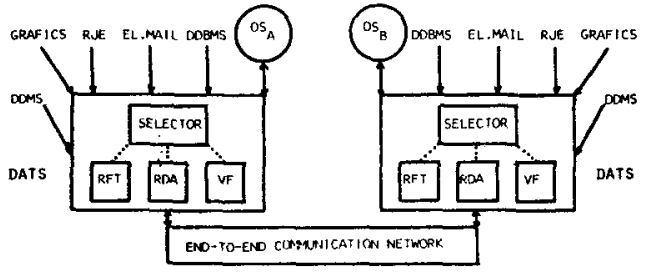

The kernel system (DATS) consists of the following components with somewhat overlapping functions:

- Remote File Transfer [5] performing the transfer of files between different file storage devices in the network.

- Remote Data Access [3] performing the access to records in files which are stored somewhere in the network.

- Virtual File performing the access to virtual data structures. The concept of virtual file is due to the fact that the HMINET will be connected to an external network with a very high degree of heterogenity. It is assumed that the file related services and transactions take place in terms of an agreed Network Virtual File, mappings being performed by local processes on to real files conform the local Data Management System. This system is at the present time in the design phase.

Since all three components may be used to perform certain similar functions, the decision of the DAT System which will be used in the execution of a user transaction is based on efficiency and performance criteria. These criteria are the result of studies based on system modelling, system simulation and computation of the protocol efficiency $[6$, $10,11,1]$

Using the above kernel a distributed graphic system, electronic mail, remote job entry and remote spool services are already implemented and further development will meet the definition of a distributed data base in the HMI-NET [12].

Also the user has a direct access to the DATS interface and can use it as the distributed data management component of the network operating system.

\section{The Data Access and Transfer System}

In addition to the user requirements, network tasks and composition, one of the design constraints of the DAT System was the need to access all the facilities of the participating data management system types.
The above constraint was imposed also by the network software design-team which has identified the data access and transfer level as a basis for most of the services the network users asked for [2].

Due to the fact the HMINET is a heterogeneous network with three different types of machines and operating systems a lot of incompatibilities have to be taken into account in the design of a Data Access and Transfer System. The following table describes the hardware and software composition of the network.

\begin{tabular}{|l|l|l|c|}
\hline COMPUTER TYPE & OS & \multicolumn{1}{|c|}{ DMS } & $\begin{array}{l}\text { NO. OF } \\
\text { INSTALLATIONS }\end{array}$ \\
\hline Siemens $7.7 \times x$ & BS 2000 & DVS & 3 \\
\hline PDP 11/40.70 & RSX11M/D & FILES*11 & 18 \\
\hline S 330 & ORG 330 & BIBEAS & 4 \\
\hline
\end{tabular}

Physically, the DAT System is made up of a combination of data processing systems which interact over a communication medium. Such interactions are controlled by distributed processes which have to accomplish tasks as different as: data link control, addressing, translation of data elements, error recovery with respect to data structure transmission, system and data resources allocation, interprocess synchronization etc.

With respect to the heterogenity and distribution of the Data Management Systems the provision of a DAT System requires [4]:

- a mechanism for selecting the needed data structure (file, record),

- a translation mechanism for the preservation of the semantic of the accessed data structure between dissimilar hosts,

- a transformation mechanism for the data structure transfer.

The DAT System in the HMINET

The following architecture of the DAT System in the HMINET was proposed and implemented: HOST A HOSI B

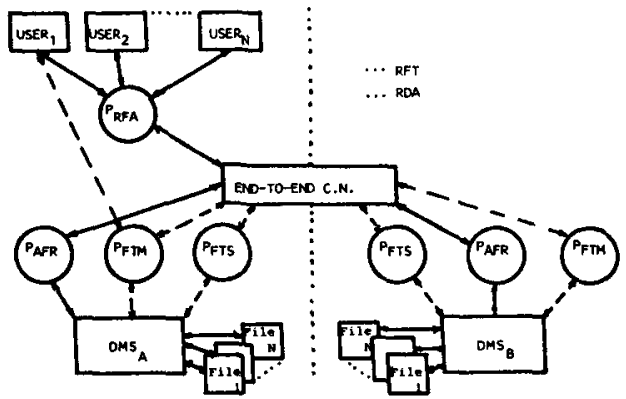


Generally, a file is considered as an identifiable whole in the network. The files are structured in units called records which represent the basic unit of information which can be accessed within a file.

The two systems: Remote File Transfer (accessing and transferring files) and Remote Data Access (accessing and transferring records) are implemented for a better reliability as two independent components. Another distinction is that the RFT is an asynchronous process, started by the user on the JCL level or by an user program, and takes place as a stand-alone process, while the record access and transfer takes place in the user program.

The access and transfer of data structures (files and records) is done by pairs of processes residing in the two hosts, for the file transfer

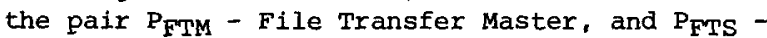
File Transfer Slave and for the Remote Data Access component the pair: $\mathrm{P}_{\mathrm{RFA}}$ (remote file access) on the usex host and one in the file residing host $P_{\text {AFR }}$ (access from remote).

A special addressing scheme in the DAT System was necessary for the link user/remote file. Each DATS process administrates local unequivocal addresses, process - user ( $P_{\text {RFA }}, P_{\text {FTM }}$ ) or process file $\left(P_{A F R}, P_{F T S}\right)$. Pairs of the above addresses define an unequivocal end-to-end connection user/ remote file (CC).

Due to the fact that both systems are symmetrically implemented an access on local files is also possible with the help of the DAT System.

The processes on the user side ( $P_{\mathrm{FTM}}, \mathrm{P}_{\mathrm{RFA}}$ ) have the following tasks:

- interpretation of the primitives and generation of the protocol elements to be sent to the partner process,

- administration and control of the local addresses (user - DAT System)

- reception, error recovery and interpretation of the protocol elements, dispatching data, control information and return codes to the users.

The processes on the host where the files reside ( $\left.P_{F T S}, P_{A F R}\right)$ perform the following major functions:

- reception and interpretation of the incoming protocol elements,

- administration and control of the local addresses of the DAT system - files and the global addresses (user-file),

- conversion of the incoming messages into local Data Management operations,

- translation of data representations,

- error recovery and generation of messages conforming to the defined protocol.
The DAT System protocol

There are several nested phases which can be identified in the proposed DAT System:

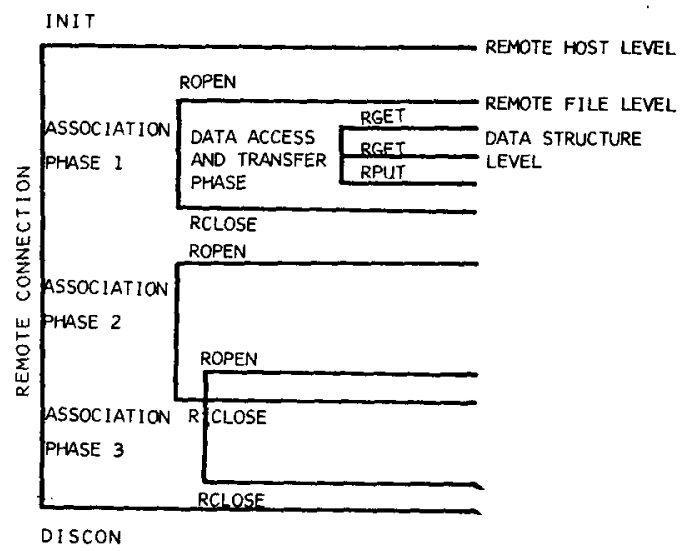

The first phase - the remote connection phase ensures the connection from user to remote host. Information about user rights on the remote host and about the resources which should be allocated and guaranteed by the DAT System for the user, the maximal common transmission buffer between the two involved processes, are the main negotiation subjects of this protocol phase.

In the next - the association phase - a connection from user to remote file is established. Several association phases are allowed during a remote connection phase depending on the allocated resources. The user rights (passwords, sharability etc.) to access a certain file are proved and a local open procedure is performed.

In the third - the data access and transfer phase - the needed data structure is read or written from/to the remote DMS.

The user program communicates with the DAT System with a set of primitives which define the frame of the DAT protocol. The following primitives are proposed to define the user/DAT System interface:

a) Initialization primitives define the remote connection phase and perform the following functions:

INIT - defines the user access rights on the remote host

- allocates a resource set in the RDA system.

(A resource set is defined as the maximum number of user-file connection which are needed by the user program and must 
be guaranteed by the DAT system at one time) .

- establishes buffer conventions between RFA and AFR.

DISCON - terminates remaining association phases

- releases the resource set

- ends the remote connection phase.

b) Control primitives belong to the association phase and operate in the following manner:

RFCB - the parameters of this primitives describe the attributes of the remote file and generate a Remote File Control Block ( $R F C B$ ) which is the information carrier between user and remote DMS

ROPEN - establishes a connection user-file (CC)

- transfers the Remote File Control Block to AFR

- prepares the remote file (local open)

RCLOSE - terminates the remote file handling

- returns information about the state of the file

- cancels the connection (CC).

The status primitive generates a temporary association phase:

RSTAT - establishes a temporary connection userfile (CC)

- returns information about the remote file

- cancels the connection.

c) Action primitives belong to the data access and transfer phase and perform the following tasks:

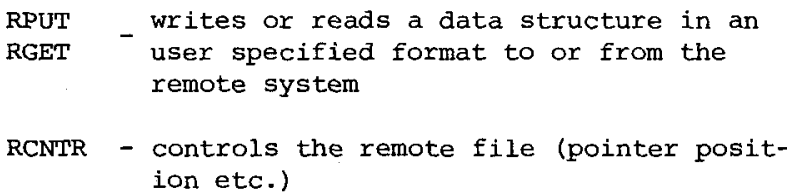

RCNTR - controls the remote file (pointer position etc.)

The files are accessed via common functions which are translated locally into functions specific to each DMS.

\section{Data Representation}

Most incompatibilities in a data access and transfer system are due to the different interpretation of physical data. Distinct data representations for each filing system lead to requirements of additional information in a DAT system in comparison with a local DMS.

A file is a particular data structure. Its elements may be organized into logical structures called records. The elements of a record are collections of bits arranged according to an explicit or implicit format as:

bits - characters - integers - real numbers.

These formats are not the same on each machine and normally a new set of physical data formats appears together with every new operating system type connected to the network.

There are two ways to solve these incompatibilities.

- declaration of a netwide standard set of formats. On every host translation from standard to the local interpretation has to be done if the standard and the local representation are not the same. Precision loss for certain systems or overhead for others must be taken into account.

- granting for all kinds of data representation present in the network.

Conversion routines must exist in every host to convert from all other data representation types into the local representation.

The source of the information "what has to be converted into what" can be:

- the user: he has to specify the format of the data elements in the record in his action primi-tives.

- the file itself: records with the internal data format specification are stored in a new type of file. Due to their new semantic these files are netwide accessable with respect to data representation. New incompatibilities between such selfdefining files and local files arise. (Programs which will locally access a self-defining file have to access it only through the DAT System.)

Comparing the above solutions we decided to permit all data representations in the network and to make the user responsible for the specification of the format of the data elements in the record. This specification is done by format parameters in the RGET and RPUT primitives.

A further development will meet the definition of a self-defining file. The influence of these development on the RDA protocol is to be found in the proposed definition of the data part in the RDA messages.

1 (DATA PART)

2 (DATA HEADER)

(DATA
(DATA)
(DATA

(DATA LENGTH)

(RECORD)

(HEADER)

8 (REP)

9 (TYPE)

The following semantics of the bytes $B$ in the above definition are assumed:

a) in the rules 3 and 5 the length in bytes and in the rule 8 the repetition factor are computed as:

first byte * $256+$ second byte 
A maximal length or repetition factor of $64 \mathrm{~K}$ can be represented.

b) in the rule $6 \mathrm{~B}$ is any bit combination in the data byte.

c) in the rule $9 \mathrm{BB}$ represents the coded data element type respective to each machine type (e.g. integer, real, character, bit string etc.).

\section{Data Transfer}

Normally, for efficiency the Data Management Systems transfer physical blocks between file supporting devices and memory. For each read or write of a record in a user program an entire block is read or written from/to device and the record handling is up to the DMS. A decrese of the response time for the DAT System must be expected on adopting this method.

This method is obvious for the Remote File Transfer component but makes sense for the Remote Data Access, too. Records are packed in a block which is transferred through the network. Due to the observation that the probability is very high for most applications to access sequential records, it seems much more efficient to implement some local record handling mechanism as an itemized transfer of records through the network.

In the actual version of the Remote Data Access protocol the above mechanism is not implemented but the influences on efficiency and costs of the protocol change are subjects of our examinations.

Another research direction with respect to the DAT System is examination of algorithms for compression and decompression of the transferred data.

\section{Conclusion and future developments}

The present paper assumes the existence of some form of transport system in the network and focuses attention upon higher level protocol design issues for a DAT system. The protocol provides mechanism for supplying arguments to remote functions and for retrieving their results; it also defines a number of data types which are used in the network environment. The set of data types is sufficient to conveniently model a large class of dat formats, but since need for additional data types will arise with new applications or new operating systems, this set remains open ende.

At the present time we can identify the DAT System as a software level in the software architecture of the computer network. Similar functions and services can be found in the MAD and DIA component, too so that future development will provide a redesign of the above two component so that the DAT System will be extended to a netwide level on top of the end-to-end leve1. A similar point of view is to be found in the ISo reference model of open systems architecture.

Future developments will meet the definitions of components in the DAT System which have to be implemented to satisfy the data access and transfer problems in computer networks with a high degree of heterogenety.

Models, simulation and efficiency studies of the design of protocol improvements will be used to support the decision of implementation.

\section{References}

[ 1] L. Kleinrock, W. E. Naylor, H. Opderbeck: A Study of Line Overhead in the ARPANET Communications of ACM Jan 76 Vol. 19 No. 1

[ 2] B. Butscher, W. L.-Bauerfeld, R. PopescuZeletin:

Data Access in Heterogeneous Computer Networks GI Symposium on "Distributed Data Bases" Proceedings

Karlsruhe, Germany, April 1978

[ 3] R. Popescu-Zeletin, B. Butscher: Specification of the RDA System in the HMINET HMI-Report B 279, Okt. 1978

[ 4$]$ S. R. Kimbleton, et al.: Network Operating System An implementation approach NCC - 1978

[ 5] W. Heinze, B. Butscher: File Transfer in the HMI Computer Network Third European Network User's Workshop IIASA, Laxenburg, Austria, April 1977

[ 6] R. Popescu-Zeletin, B. Butscher: A Study of Efficiency and overhead of Data Access and Transfer Systems in the Heterogeneous Computer Network German Chapter of ACM Workshop on Communication Networks, Wiesbaden, Germany, June 1978

[ 7] G. E. White: A High-level Framework for network-based Resource Sharing NCC - 1976

[ 8] W. L.-Bauerfeld, H. Strack-Zimmerman: The Hahn-Meitner-Institut Computer Network Workshop on Data Communication, IIASA, Austria, Sept. 1978

[ 9] M. Gien: Proposal for a standard File Transfer Protocol (FTP)

DAT 519 EIN/IRIA/77/4, MaY 1977

[10] K. M. Chandy, D. Baum, W. L.-Bauerfela, R. Popescu-Zeletin, K. Ullmann: Data flow analysis for fiele transfer and remote data access protocols - to be published - 
[11] D. Baum, W. L.-Bauerfeld, R. Popescu-Zeletin, K. Ullmann:

End-to-End level data flow analysis for communication networks

Proceedings of the International Symposium on

Flow Control in Computer Networks

Versailles, France, Feb. 12-14, 1979

[12] R. Popescu-zeletin, B. Butscher: Access Systems for a Distributed Data Base

Proceedings of the International Congress on

Data Processing

Berlin, West-Germany, Sept. 1978 